\title{
Genetic Analysis of Species in the Genus Catasetum (ORCHIDACEAE) using RAPD Markers
}

\author{
Luciana do Valle Rego Oliveira ${ }^{1}$, Ricardo Tadeu de Faria ${ }^{*}$, Claudete de Fátima Ruas ${ }^{2}$, \\ Paulo Maurício Ruas ${ }^{2}$, Melissa de Oliveira Santos ${ }^{2}$ and Valdemar P. Carvalho ${ }^{2}$ \\ ${ }^{1}$ Departamento de Agronomia; Universidade Estadual de Londrina; Celso Garcia Cid Km 379; 86051-990; \\ Londrina - PR - Brasil. ${ }^{2}$ Departamento de Biologia Geral; Universidade Estadual de Londrina; Celso Garcia Cid \\ Km 379; 86051-990; Londrina - PR - Brasil
}

\begin{abstract}
In this work, RAPD molecular markers were used to access the genetic variability and to study the inter and intraespecifc relationship in a group of 37 species, including 56 individuals. A total of 15 RAPD primers were selected for DNA amplification. From a total of 221 bands analyzed, 209 (95\%) were polymorphics. The level of interespecifc genetic similarity ranged from $37 \%$ between Catasetum complanatum and Catasetum laminatum to $83 \%$ between Catasetum triodon and Catasetum uncatum. The intraspecifc genetic similarity varied $88 \%$ for the individuals of Catasetum triodon to $93 \%$ between the individuals of Catasetum atratum and Catasetum macrocarpum. These results would contribute to understand the genetic relationship in Catasetum, to define the strategies to establish a germplasm core collection for the genus and to provide support for breeding programs.
\end{abstract}

Key words: Catasetum, molecular marker, orchid, RAPD, genetic similarity

\section{INTRODUCTION}

The family Orchidaceae has unique characteristics that do not resemble with any other group of plants (Rittershausen, 1998). It is considered the largest family within the group of flowering plants, and comprises approximately 35,000 species distributed across six tribes, 80 subtribes, and about 750 genera (Heywood, 1993). It constitutes a very successful group, with more than 100,000 hybrids developed in the last 150 years, both by natural and artificial crosses (Suttleworth et al., 1993; Rittershausen, 1998; Faria et al., 2001; Moreira and Isaias, 2008).

The classification of the family Orchidaceae is not yet well defined. The classification system has been developed for centuries and was established mainly by taxonomists. It includes a number of divisions and subdivisions that group the plants based on their similarities in structure and flower appearance. The most accepted classification at present takes into account other plant characteristics, such as cytological and molecular data (Dressler, 1993).

The genus Catasetum has more than 100 species (Raposo, 1992), of which the major part is terrestrial or epiphytic (Scaglia, 1998). The habitat of this genus extends from Mexico down to Argentina, with a center of radiation in Brazil (Raposo, 1992). It includes some of the most beautiful and rare species (ABRACC, 1998a). The plant considered as typical in the genus Catasetum

*Author for correspondence: faria@uel.br 
is Catasetum macrocarpum. Other synonyms of the genus that were often used in the past are: Catachaeteum Hoffmansegg. 1842; Cuculina Raf. 1836; Monacanthus G. Don 1839; Monachanthus Lindley 1832; Myanthus Lindley 1832; and Warczewitzia Skinner $1850 \quad$ (Orchidaceae brasiliensis, 2004).

The genus Catasetum, as well as the majority of the family Orchidaceae, presents chromosomal variation, such as: $n=27,28,54$, and 81 (Tanaka and Kamemoto, 1984). However, for some genera, the basic number of chromosomes is usually constant: 20 in Cattleya, 15 in Vanda, 19 in Dendrobium and Phalaenopsis (Carnier, 1996). Occasionally, changes in the number of chromosomes do occur, resulting in an increase in the degree of ploidy. The increase in ploidy in orchids is often accompanied by an increase in the size of plant parts; plants are more robust and flowers generally have a better shape, and could become gigantic (Carnier, 1996). According to Tanaka and Kamemoto (1984), chromosome number determination is an important tool to conduct the breeding studies in this family.

Molecular markers have been widely used in genetic diversity studies, and also as an aid in the classification of orchid species and genera (Tsai et al., 2002; Obara-Okeyo and Kako, 1998; Williams et al, 2001).

Many studies have tried to establish a molecular phylogeny in the family Orchidaceae. Freudenstein and Doyle (1994) used chloroplast DNA as part of a phylogenetic study in Corallorhiza, while Squirrel et al. (2001) used chloroplast genes and the variability obtained by isozymes in different Epipactis helleborine populations. Ribosomal ITS sequences have been employed in other studies, either individually or together with sequences to make inferences about interspecific relationships in different groups of orchids (Gravendeel et al., 2001; Pridgeon et al., 2001; Williams et al., 2001).

The RAPD technique (Random Amplified Polymorphic DNA) has been used in certain orchid groups in order to study their structure and genetic diversity. Tsai et al. (2002) studied several genotypes in the Tribe Oncidiinae using RAPD markers and revealed a genetic similarity that ranged between 25 and $71 \%$. Obara - Okeyo and Kako (1998) used 132 RAPD markers to analyze the efficacy of this technique in the identification of Cymbidium cultivars. Their results showed that it was possible to discriminate all the cultivars when an adequate quantity of primers was used. In other studies, RAPD markers were used in conjunction with isozymes, in order to analyze the genetic structure and conservation of orchid populations. A correlation between data obtained with the use of both types of markers was observed (Case et al., 1998; Sun and Wong, 2001). The objective of this work was to determine the genetic similarity between 37 species in the genus Catasetum, including 56 individuals, and to clarify the intra and interspecific relationships in this genus using RAPD molecular markers.

\section{MATERIAL AND METHODS}

\section{Plant material}

The plant material was obtained from the orchid collection of the Agronomy Department at Centro de Ciências Agrárias of Universidade Estadual de Londrina, in Brazil. The species and subspecies studied, as well as their corresponding names in the collection, chromosome numbers (2n), general geographical distribution and specific origin (Table $1)$.

\section{DNA extraction}

Genomic DNA was extracted following the methodology described by Doyle and Doyle (1987), with some modifications. DNA was extracted from young leaves of individual plants macerated in liquid nitrogen, and mixed with 2.5 $\mathrm{mL}$ of $3 \%$ CTAB extraction buffer (3\% CTAB; $150 \mathrm{mM}$ Tris - HCL; $30 \mathrm{mM}$ EDTA, ph 7.5; 2.2 $\mathrm{mM} \mathrm{NaCl}, \quad 1.5 \% \quad \mathrm{PVP})$ and $4 \mu \mathrm{L} \quad \beta \quad-$ mercaptoethanol. The samples were then incubated in a double boiler at $65^{\circ} \mathrm{C}$ for 30 minutes and centrifuged for five minutes at $14,000 \mathrm{rpm}$. After centrifugation, the supernatant was isolated and 1.5 volume of isopropanol was added, followed by incubation at $-20^{\circ} \mathrm{C}$ for 30 minutes. At the end of incubation, the samples were submitted to centrifugation for five minutes at $14,000 \mathrm{rpm}$ and then the supernatant was discarded. The resulting precipitate was washed twice in $70 \%$ alcohol, resuspended in $0.1 \mathrm{~mL} \mathrm{TE}$ buffer (Tris-HCl $1 \mathrm{mM}$; EDTA $0.1 \mathrm{mM}, \mathrm{pH} 8.0$ ) and added of $1 \mu \mathrm{l}$ proteinase $\mathrm{K}(10 \mathrm{mg} / \mu \mathrm{L})$. After incubation for one hour at $37^{\circ} \mathrm{C}$, one volume of $3 \mathrm{M}$ ammonia acetate was added and the samples were incubated on ice for 20 minutes. After this 
period, the same volume of isopropanol wasadded, followed by incubation for at least 20 minutes at $20^{\circ} \mathrm{C}$. The DNA was recovered by centrifugation at $14,000 \mathrm{rpm}$ for five minutes, washed in $70 \%$ alcohol and dissolved in TE buffer. The DNA was quantified with a fluorometer (Dyna-Quant, Hoefer B Pharmacia) and diluted to a final concentration of $10 \mathrm{ng} / \mu \mathrm{l}$.

Table 1 - Species in the genus Catasetum, subspecies, collection, number of chromosomes (2n), geographical distribution, and origin of species studied by means of the RAPD technique.

\begin{tabular}{|c|c|c|c|c|c|c|}
\hline$\frac{\mathbf{n}^{0}}{n^{0}}$ & Genus & species & subspecies & Collection & Geographical Distribution & Origin of the Species under Study \\
\hline 1 & Ctsm. & arietinum & & Hideto Ctsm. 2 & $\mathrm{PE}$ & $\mathrm{PE}$ \\
\hline 2 & Ctsm. & aripuanense & & UEL Ctsm. 37 & MS and MT & MT \\
\hline 3 & Ctsm. & ariquimense & & Igor Ctsm. 6 & RO & RO \\
\hline 4 & Ctsm. & atratum & & UEL Ctsm. 11 e 24 & SP, PR, SC, RS and MG & PR \\
\hline 5 & Ctsm. & atratum & & UEL Ctsm. 66 & $\mathrm{SP}, \mathrm{PR}, \mathrm{SC}, \mathrm{RS}$ and $\mathrm{MG}$ & Unknown origin \\
\hline 6 & Ctsm. & atratum & & UEL Ctsm. 58 & $\mathrm{SP}, \mathrm{PR}, \mathrm{SC}, \mathrm{RS}$ and $\mathrm{MG}$ & Unknown origin \\
\hline 7 & Ctsm. & barbatum & semicuculatum & UEL Ctsm. 3 & TO, PA, AM, AC, MT, MA, MS, CE and PI & Unknown origin \\
\hline 8 & Ctsm. & barbatum & spinosum & UEL Ctsm. 10 e 12 & TO, PA, AM, AC, MT, MA, MS, CE and PI & Unknown origin \\
\hline 9 & Ctsm. & barbatum & & UEL Ctsm. 26 Hideto Ctsm. 3 & TO, PA, AM, AC, MT, MA, MS, CE and PI & Unknown origin \\
\hline 10 & Ctsm. & сегnиит & & UEL Ctsm. 47 & $\mathrm{SP}, \mathrm{RJ}, \mathrm{PR}, \mathrm{ES}$ and MG & RJ \\
\hline 11 & Ctsm. & ciliatum & & UEL Ctsm. 63 & $\mathrm{PA}$ and $\mathrm{AM}$ & PA \\
\hline 12 & Ctsm. & complanatum & & UEL Ctsm. 6 e 40 & RO & RO \\
\hline 13 & Ctsm. & confusum & & Igor Ctsm. 2 & GO & GO \\
\hline 14 & Ctsm. & cristatum & & UEL. Ctsm. 34 & $\mathrm{RR}, \mathrm{AP}$ and $\mathrm{PA}$ & Unknown origin \\
\hline 15 & Ctsm. & denticulatum & & Igor Ctsm. 1 & RO & RO \\
\hline 16 & Ctsm. & discolor & & UEL. Ctsm. 32 e 35 & $\begin{array}{l}\text { AM, PA, MA, CE, MT, PE, BA, ES, EJ, } \\
\text { Suriname and Guianas }\end{array}$ & BA \\
\hline 17 & Ctsm. & discolor & & UEL. Ctsm. 27 e 22 & $\begin{array}{l}\text { AM, PA, MA, CE, MT, PE, BA, ES, EJ, } \\
\text { Suriname and Guianas }\end{array}$ & $\mathrm{PA}$ \\
\hline 18 & Ctsm. & fimbriatum & & UEL. Ctsm. 30 e 42 & MT, MS, SP, PR, MG and GO & MG \\
\hline 19 & Ctsm. & fimbriatum & ornithorhynchum & UEL Ctsm. 49 & MT, MS, SP, PR, MG and GO & Unknown origin \\
\hline 20 & Ctsm. & fimbriatum & & UEL Ctsm. 56 e 64 & MT, MS, SP, PR, MG and GO & Unknown origin \\
\hline 21 & Ctsm. & fimbriatum & fissum & UEL Ctsm. 23 & MT, MS, SP, PR, MG and GO & PR \\
\hline 22 & Ctsm. & fimbriatum & (flor vermelha) & UEL Ctsm. 67 & MT, MS, SP, PR, MG and GO & Unknown origin \\
\hline 23 & Ctsm. & galeritum & & Hideto Ctsm.4 & $\mathrm{AM}, \mathrm{PA}, \mathrm{GO}$ and TO & Unknown origin \\
\hline 24 & Ctsm. & gardneri & & UEL Ctsm. 5 e 19 & PE to RJ & $\mathrm{BA}$ \\
\hline 25 & Ctsm. & gladiatorium & & UEL Ctsm. 9 & GO and MT(Brasil's central plateau) & GO \\
\hline 26 & Ctsm. & gladiatorium & & UEL Ctsm. 62 & GO and MT(Brasil's central plateau) & Unknown origin \\
\hline 27 & Ctsm. & gnomus & & UEL Ctsm. 45 e 31 & $\mathrm{AM}$ & $\mathrm{AM}$ \\
\hline 28 & Ctsm. & hookeri & & UEL Ctsm. 39 & SP and RJ & Unknown origin \\
\hline 39 & Ctsm. & hookeri & & UEL Ctsm. 36 e 53 & SP and RJ & Unknown origin \\
\hline 30 & Ctsm. & juruenense & & UEL Ctsm. 38 & MT & MT \\
\hline 31 & Ctsm. & juruenense & & UEL Ctsm. 28 & MT & MT \\
\hline 32 & Ctsm. & laminatum & & UEL Ctsm. 51 & México & Unknown origin \\
\hline 33 & Ctsm. & lemosii & & Igor $C t s m .5$ & PAand MT & Unknown origin \\
\hline 34 & Ctsm. & macrocarpum & & UEL Ctsm. 2 & $\begin{array}{c}\text { RJ, BA, PB, PE, RN, PI, PA, AM, TO, MA, } \\
\text { RR and ES }\end{array}$ & $\mathrm{PA}$ \\
\hline 35 & Ctsm. & macrocarpum & & UEL Ctsm. 25 e 29 & $\begin{array}{c}\text { RJ, BA, PB, PE, RN, PI, PA, AM, TO, MA, } \\
\text { RR and ES }\end{array}$ & BA \\
\hline 36 & Ctsm. & macrocarpum & & UEL Ctsm. 41 & $\begin{array}{c}\text { RJ, BA, PB, PE, RN, PI, PA, AM, TO, MA, } \\
\text { RR and ES }\end{array}$ & Unknown origin \\
\hline 37 & Ctsm. & matogrossense & & Igor Ctsm. 11 & MT & MT \\
\hline 38 & Ctsm. & moreanum & & Igor Ctsm. 9 & Brazil & BA \\
\hline 39 & Ctsm. & osculatum & & UEL Ctsm. 13 & MS, MT, RO and GO & MT \\
\hline 40 & Ctsm. & osculatum & & UEL Ctsm. 55 & MS, MT, RO and GO & MS \\
\hline 41 & Ctsm. & osculatum & & UEL Ctsm. 20 & MS, MT, RO and GO & RO \\
\hline 42 & Ctsm. & parguazense & & Igor Ctsm. 12 & RO & RO \\
\hline 43 & Ctsm. & pileatum & & UEL Ctsm.68 & $\mathrm{AM}$ & Unknown origin \\
\hline 44 & Ctsm. & planiceps & & Igor $C t s m .8$ & $\begin{array}{c}\text { AM (norte do Brasil), Venezuela, Guianas, } \\
\text { Suriname e Perú }\end{array}$ & PA \\
\hline 45 & Ctsm. & pulchorom & & Hideto Ctsm. 5 & $\mathrm{AM}, \mathrm{PA}$ and $\mathrm{MT}$ & MT \\
\hline 46 & Ctsm. & purum & & UEL Ctsm. 16 e 43 & BA, ES and MG & BA \\
\hline 47 & Ctsm. & saccatum & & UEL Ctsm. 65 & $\mathrm{AC}$ and $\mathrm{AM}$ & Unknown origin \\
\hline 48 & Ctsm. & sanguineum & & UEL Ctsm. 4 & $\begin{array}{l}\text { Colombia, Guiana, Venezuela, Brazil, Costa } \\
\text { Rica and Panama }\end{array}$ & $\mathrm{AC}$ \\
\hline 49 & Ctsm. & schmidtianum & & Igor Ctsm. 4 & MT & MT \\
\hline 50 & Ctsm. & spitzii & & UEL Ctsm. 18,54 e 59 & GO & Unknown origin \\
\hline 51 & Ctsm. & spitzii & & UEL Ctsm. 14 & GO & Unknown origin \\
\hline 52 & Ctsm. & triodom & & UEL Ctsm. 8 e 57 & SP, PR and SC & PR \\
\hline 53 & Ctsm. & triodom & & UEL Ctsm. 33 & SP, PR and SC & PR \\
\hline 54 & Ctsm. & uncatum & & UEL Ctsm. 21,61 e 50 & $\mathrm{PE}$ & Unknown origin \\
\hline 55 & Ctsm. & vinaceum & & $\begin{array}{l}\text { UEL Ctsm. } 15,60 \text { e } 48 \\
\text { Hideto Ctsm. } 6\end{array}$ & MT, MS and GO & MT \\
\hline 56 & Ctsm. & vinaceum & & UEL Ctsm. 1 & MT, MS and GO & Unknown origin \\
\hline
\end{tabular}


DNA amplification via PCR (Polymerase Chain Reaction)

The RAPD reactions were conducted in a final volume of $15 \mu \mathrm{l}$ containing $7.92 \mu \mathrm{l}$ sterile water; $1.5 \mu \mathrm{l}$ amplification buffer $(50 \mathrm{mM} \mathrm{KCl}, 15 \mathrm{mM}$ $\mathrm{MgCl}_{2}, 100 \mathrm{mM}$ Tris-Cl, pH 9.0); $1.8 \mu \mathrm{l}$ dNTPs (0.1 mM of each dGTP, dATP, dCTP, dTTP); 1.8 $\mu$ primer -Operon Technologies (4 mM); 1U Taq DNA polymerase (Biotools); and $20 \mathrm{ng}$ DNA (polymerization), followed by a final extension for $7 \mathrm{~min}$ at $72^{\circ} \mathrm{C}$. The amplification products were separated by electrophoresis in $1 \%$ agarose gel, stained with ethidium bromide $(0.15 \mu \mathrm{l} / \mathrm{ml})$, and visualized under UV light. Gel images were captured using a photographic documentation and thermoprinting system.

\section{Data analysis}

The amplification products were analyzed by marking their presence (1) or absence (0) for each DNA fragment generated. The data obtained were analyzed with the NTSYS-PC software (Rohlf,
2000) and utilized to obtain the genetic similarity matrix using DICE coefficient. The UPGMA clustering method (Unweighted Pair Group Method using Arithmetic Averages) was used to construct a dendrogram. The reliability of the associations shown on the dendrogram was evaluated by bootstrap probability with 1,000 permutations, using the bood v. 3.0 software (Coelho 2001). The cophenetic coefficient between the similarity matrix and the dendrogram was computed using the NTSYS program.

\section{RESULTS AND DISCUSSION}

A total of 15 RAPD primers (Table 2) were selected and used for DNA amplification. Two hundred and twenty-one markers were generated, of which 209 were polymorphic (95\%). The electrophoretic pattern obtained with primer OPAE - 16 is shown in Figure 1.

Table 2 - Primers used sequence of primers (Operon Technologies), number of bands, and percentage of polymorphic bands for species in the genus Catasetum under study.

\begin{tabular}{cccc}
\hline Primer & Primer Sequence & Number of bands & $\begin{array}{c}\text { Clustered polymorphic } \\
\text { bands }\end{array}$ \\
\hline OPAW-01 & 5' ACCTAGGGGA 3' & 6 & $83 \%$ \\
OPAW-03 & 5' CCATGCGGAG 3' & 20 & $90 \%$ \\
OPAW-05 & 5' CTGCTTCGAG 3' & 21 & $95 \%$ \\
OPAW-10 & 5' GGTGTTTGCC 3' & 14 & $86 \%$ \\
OPAW-15 & 5' CCAGTCCCAA 3' & 14 & $93 \%$ \\
OPAE-16 & 5' TCCGTGCTGA 3' & 19 & $100 \%$ \\
OPAI-01 & 5' GGCATCGGCT 3' & 8 & $88 \%$ \\
OPAI-02 & 5' AGCCGTTCAG 3' & 15 & $92 \%$ \\
OPAD-07 & 5' CCCTACTGGT 3' & 16 & $93 \%$ \\
OPAD-15 & 5' TTTGCCCCGT 3' & 23 & $96 \%$ \\
OPAD-13 & 5' GGTTCCTCTG 3' & 17 & $100 \%$ \\
OPAD-19 & 5' CTTGGCACGA 3' & 12 & $92 \%$ \\
OPAS-18 & 5' GTTGCGCAGT 3' & 20 & $100 \%$ \\
OPAX-04 & 5' TCCCCAGGAG 3' & 8 & $100 \%$ \\
OPAX-07 & 5' ACGCGACAGA 3' & 9 & $100 \%$ \\
\hline Total & & 251 & $95 \%$ \\
\hline
\end{tabular}

The RAPD data were used to generate a similarity matrix (Table 3) and the dendrogram (Fig. 2). In the dendrogram, most associations between groups obtained bootstrap values (BO) higher than $50 \%$, thus attesting to the reliability of this experiment.

The polymorphism generated with RAPD markers allowed the species under analysis to be divided into several distinct groups. The high value obtained in the cophenetic analysis $(r=0.922)$ ensured the reliability of the clusters observed in the dendrogram. The significance of the correlation, tested according to Mantel's test, showed that the number of markers used was sufficient to assess the genetic diversity of the species analyzed. 

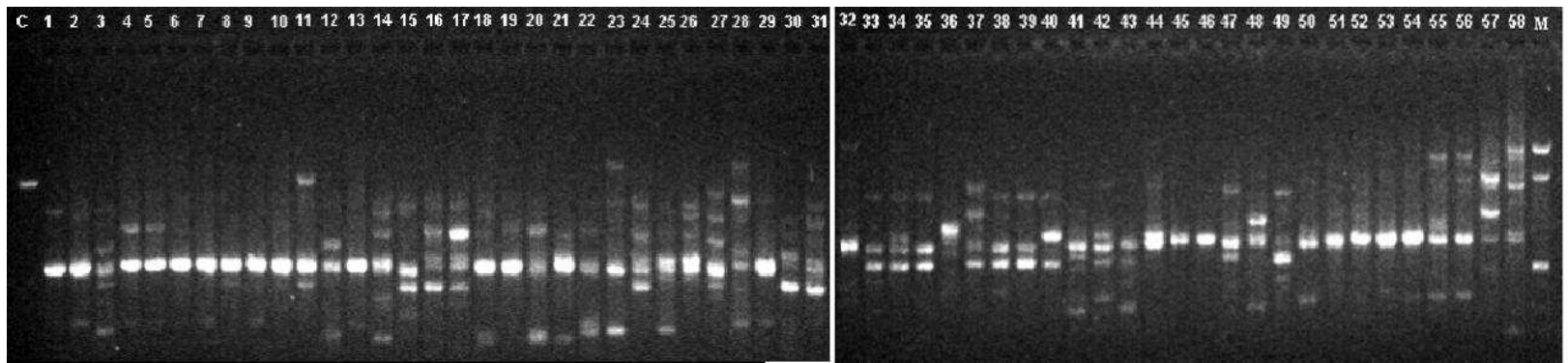

Figure 1 - Electrophoretic pattern obtained with primer OPAE - 16. Samples are identified by the numbers presented in Table 2. M - Molecular weight marker (DNA ladder Invitrogen 100pb), C - Control sample.

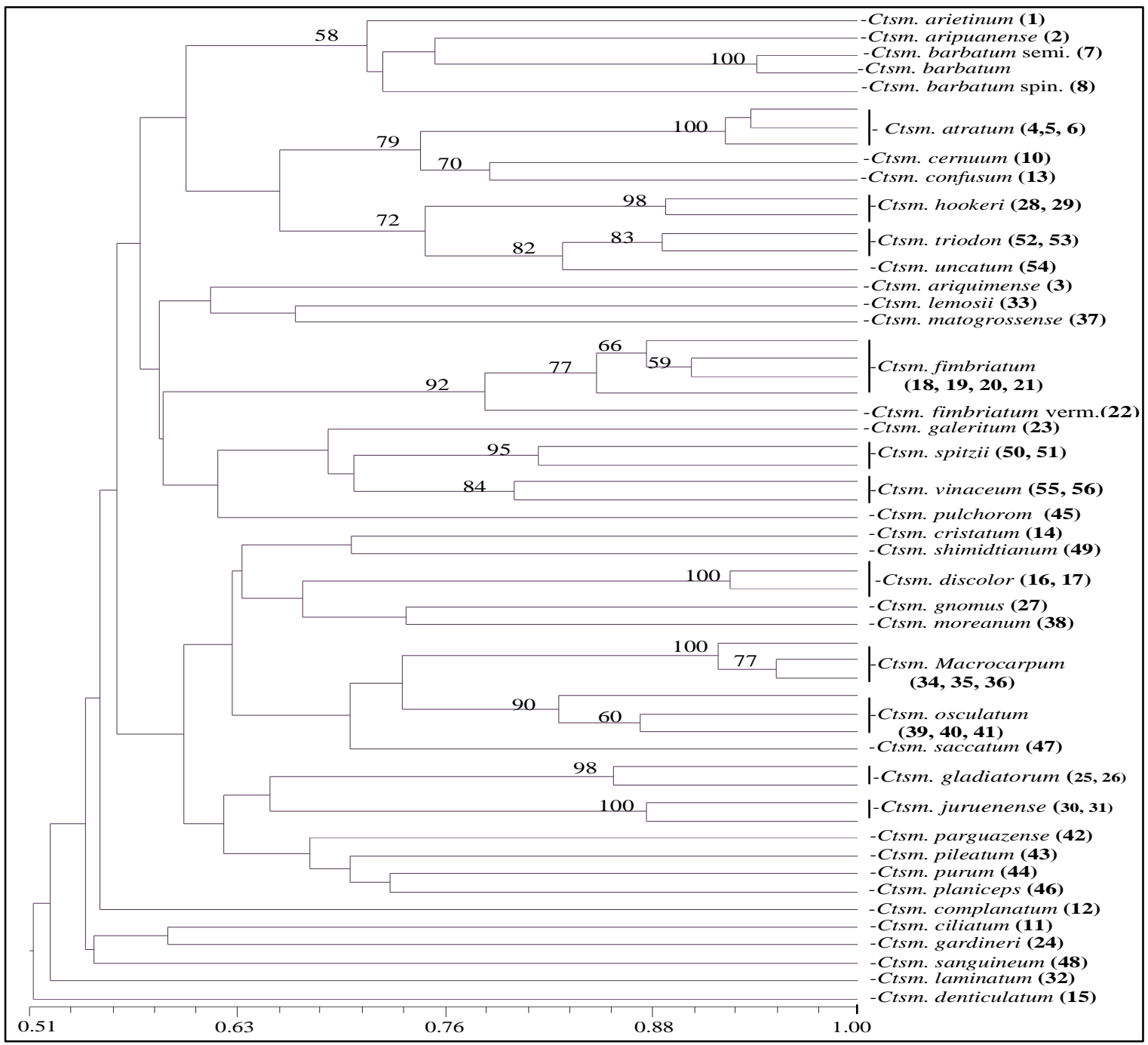

Figure 2 - Genetic similarity dendrogram of 37 species studied in the Genus Catasetum, including 56 individuals, calculated by DICE index, based on RAPD markers. The number between parentheses corresponds to the sequence presented in table 1. Bootstrap values below 50\% are not shown. Londrina (PR, Brazil), UEL, 2004. 
Table 3 - Genetic similarity matrix obtained for 37 species of Catasetum with 56 individuals obtained from RAPD markers. Londrina (PR, Brazil), UEL, 2004.

\begin{tabular}{|c|c|c|c|}
\hline Ctsm. & 1 & 2 & 3 \\
\hline 1 & 1,00 & & \\
\hline 2 & 0,74 & 1,00 & \\
\hline 3 & 0,64 & 0,60 & 1,00 \\
\hline 4 & 0,67 & 0,68 & 0,56 \\
\hline 5 & 0,63 & 0,65 & 0,54 \\
\hline 6 & 0,64 & 0,63 & 0,56 \\
\hline 7 & 0,71 & 0,75 & 0,52 \\
\hline 8 & 0,68 & 0,66 & 0,59 \\
\hline 9 & 0,70 & 0,75 & 0,51 \\
\hline 10 & 0,62 & 0,62 & 0,54 \\
\hline 11 & 0,45 & 0,53 & 0,49 \\
\hline 12 & 0,58 & 0,54 & 0,52 \\
\hline 13 & 0,67 & 0,66 & 0,55 \\
\hline 14 & 0,60 & 0,61 & 0,58 \\
\hline 15 & 0,51 & 0,51 & 0,58 \\
\hline 16 & 0,54 & 0,52 & 0,59 \\
\hline 17 & 0,54 & 0,50 & 0,59 \\
\hline 18 & 0,61 & 0,54 & 0,57 \\
\hline 19 & 0,59 & 0,57 & 0,57 \\
\hline 20 & 0,65 & 0,60 & 0,57 \\
\hline 21 & 0,65 & 0,59 & 0,59 \\
\hline 22 & 0,60 & 0,55 & 0,58 \\
\hline 23 & 0,60 & 0,66 & 0,60 \\
\hline 24 & 0,56 & 0,54 & 0,53 \\
\hline 25 & 0,60 & 0,59 & 0,61 \\
\hline 26 & 0,58 & 0,61 & 0,57 \\
\hline 27 & 0,59 & 0,58 & 0,60 \\
\hline 28 & 0,57 & 0,59 & 0,55 \\
\hline 29 & 0,58 & 0,61 & 0,54 \\
\hline 30 & 0,55 & 0,52 & 0,56 \\
\hline 31 & 0,58 & 0,56 & 0,60 \\
\hline 32 & 0,53 & 0,53 & 0,48 \\
\hline 33 & 0,66 & 0,64 & 0,58 \\
\hline 34 & 0,61 & 0,57 & 0,56 \\
\hline 35 & 0,61 & 0,52 & 0,55 \\
\hline 36 & 0,61 & 0,53 & 0,54 \\
\hline 37 & 0,59 & 0,61 & 0,65 \\
\hline 38 & 0,57 & 0,53 & 0,53 \\
\hline 39 & 0,62 & 0,56 & 0,57 \\
\hline 40 & 0,62 & 0,57 & 0,58 \\
\hline 41 & 0,58 & 0,56 & 0,57 \\
\hline 42 & 0,63 & 0,52 & 0,57 \\
\hline 43 & 0,62 & 0,53 & 0,55 \\
\hline 44 & 0,59 & 0,58 & 0,56 \\
\hline 45 & 0,50 & 0,57 & 0,53 \\
\hline 46 & 0,59 & 0,56 & 0,56 \\
\hline 47 & 0,53 & 0,55 & 0,50 \\
\hline 48 & 0,53 & 0,54 & 0,55 \\
\hline 49 & 0,60 & 0,55 & 0,66 \\
\hline 50 & 0,57 & 0,56 & 0,52 \\
\hline 51 & 0,53 & 0,54 & 0,55 \\
\hline 52 & 0,59 & 0,59 & 0,47 \\
\hline 53 & 0,58 & 0,58 & 0,52 \\
\hline 54 & 0,61 & 0,55 & 0,55 \\
\hline 55 & 0,63 & 0,61 & 0,60 \\
\hline 56 & 0,62 & 0,59 & 0,56 \\
\hline Ctsm. & 20 & 21 & 22 \\
\hline 20 & 1,00 & & \\
\hline 21 & 0,87 & 1,00 & \\
\hline 22 & 0,76 & 0,81 & 1,00 \\
\hline 23 & 0,60 & 0,61 & 0,64 \\
\hline 24 & 0,55 & 0,51 & 0,50 \\
\hline 25 & 0,64 & 0,64 & 0,70 \\
\hline 26 & 0,68 & 0,63 & 0,63 \\
\hline 27 & 0,65 & 0,67 & 0,69 \\
\hline 28 & 0,57 & 0,58 & 0,57 \\
\hline 29 & 0,56 & 0,59 & 0,54 \\
\hline 30 & 0,52 & 0,53 & 0,56 \\
\hline 31 & 0,51 & 0,57 & 0,60 \\
\hline 32 & 0,47 & 0,52 & 0,49 \\
\hline 33 & 0,65 & 0,63 & 0,62 \\
\hline 34 & 0,57 & 0,61 & 0,61 \\
\hline 35 & 0,58 & 0,62 & 0,61 \\
\hline 36 & 0,58 & 0,62 & 0,63 \\
\hline 37 & 0,57 & 0,55 & 0,59 \\
\hline 38 & 0,52 & 0,50 & 0,56 \\
\hline 39 & 0,59 & 0,59 & 0,60 \\
\hline 40 & 0,60 & 0,59 & 0,62 \\
\hline 41 & 0,53 & 0,57 & 0,59 \\
\hline 42 & 0,55 & 0,57 & 0,62 \\
\hline 43 & 0,49 & 0,56 & 0,64 \\
\hline 44 & 0,54 & 0,57 & 0,67 \\
\hline 45 & 0,53 & 0,55 & 0,53 \\
\hline 46 & 0,47 & 0,53 & 0,54 \\
\hline 47 & 0,54 & 0,59 & 0,60 \\
\hline 48 & 0,54 & 0,48 & 0,50 \\
\hline 49 & 0,57 & 0,60 & 0,61 \\
\hline 50 & 0,58 & 0,61 & 0,58 \\
\hline 51 & 0,57 & 0,57 & 0,57 \\
\hline 52 & 0,56 & 0,55 & 0,51 \\
\hline 53 & 0,51 & 0,50 & 0,47 \\
\hline 54 & 0,55 & 0,56 & 0,53 \\
\hline 55 & 0,64 & 0,57 & 0,64 \\
\hline 56 & 0,64 & 0,59 & 0,65 \\
\hline
\end{tabular}


(Cont. Table 3)

\begin{tabular}{|c|c|c|c|c|c|c|c|c|c|c|c|c|c|c|c|c|c|c|}
\hline Gsm & 39 & 40 & 41 & 42 & 43 & 44 & 45 & 46 & 47 & 48 & 49 & 50 & 51 & 52 & 53 & 54 & 55 & 56 \\
\hline 39 & 1,00 & & & & & & & & & & & & & & & & & \\
\hline 40 & 0,86 & 1,00 & & & & & & & & & & & & & & & & \\
\hline 41 & 0,78 & 0,87 & 1,00 & & & & & & & & & & & & & & & \\
\hline 42 & 0,62 & 0,62 & 0,59 & 1,00 & & & & & & & & & & & & & & \\
\hline 43 & 0,73 & 0,68 & 0,65 & 0,67 & 1,00 & & & & & & & & & & & & & \\
\hline 44 & 0,68 & 0,66 & 0,69 & 0,70 & 0,70 & 1,00 & & & & & & & & & & & & \\
\hline 45 & 0,57 & 0,59 & 0,62 & 0,61 & 0,53 & 0,62 & 1,00 & & & & & & & & & & & \\
\hline 46 & 0,60 & 0,62 & 0,63 & 0,66 & 0,69 & 0,72 & 0,59 & 1,00 & & & & & & & & & & \\
\hline 47 & 0,66 & 0,68 & 0,72 & 0,58 & 0,60 & 0,68 & 0,63 & 0,65 & 1,00 & & & & & & & & & \\
\hline 48 & 0,57 & 0,58 & 0,57 & 0,60 & 0,52 & 0,61 & 0,60 & 0,57 & 0,60 & 1,00 & & & & & & & & \\
\hline 49 & 0,66 & 0,64 & 0,65 & 0,62 & 0,68 & 0,65 & 0,56 & 0,62 & 0,66 & 0,61 & 1,00 & & & & & & & \\
\hline 50 & 0,58 & 0,60 & 0,59 & 0,60 & 0,55 & 0,52 & 0,60 & 0,56 & 0,56 & 0,54 & 0,53 & $1, \infty 0$ & & & & & & \\
\hline 51 & 0,55 & 0,59 & 0,56 & 0,59 & 0,51 & 0,52 & 0,61 & 0,57 & 0,52 & 0,53 & 0,50 & 0,81 & 1,00 & & & & & \\
\hline 52 & 0,56 & 0,55 & 0,56 & 0,54 & 0,51 & 0,53 & 0,63 & 0,59 & 0,53 & 0,53 & 0,50 & 0,66 & 0,61 & 1,00 & & & & \\
\hline 53 & 0,56 & 0,55 & 0,57 & 0,56 & 0,54 & 0,52 & 0,64 & 0,60 & 0,51 & 0,54 & 0,51 & 0,61 & 0,66 & 0,88 & 1,00 & & & \\
\hline 54 & 0,57 & 0,56 & 0,57 & 0,59 & 0,53 & 0,55 & 0,64 & 0,65 & 0,54 & 0,56 & 0,54 & 0,67 & 0,69 & 0,80 & 0,85 & 1,00 & & \\
\hline 55 & 0,60 & 0,63 & 0,60 & 0,62 & 0,56 & 0,60 & 0,65 & 0,57 & 0,53 & 0,58 & 0,59 & 0,73 & 0,71 & 0,70 & 0,67 & 0,75 & 1,00 & \\
\hline 56 & 0,59 & 0,59 & 0,52 & 0,63 & 0,54 & 0,52 & 0,59 & 0,53 & 0,53 & 0.57 & 0,55 & 0,70 & 0,67 & 0,60 & 0,57 & 0,65 & 0,80 & 1,00 \\
\hline
\end{tabular}

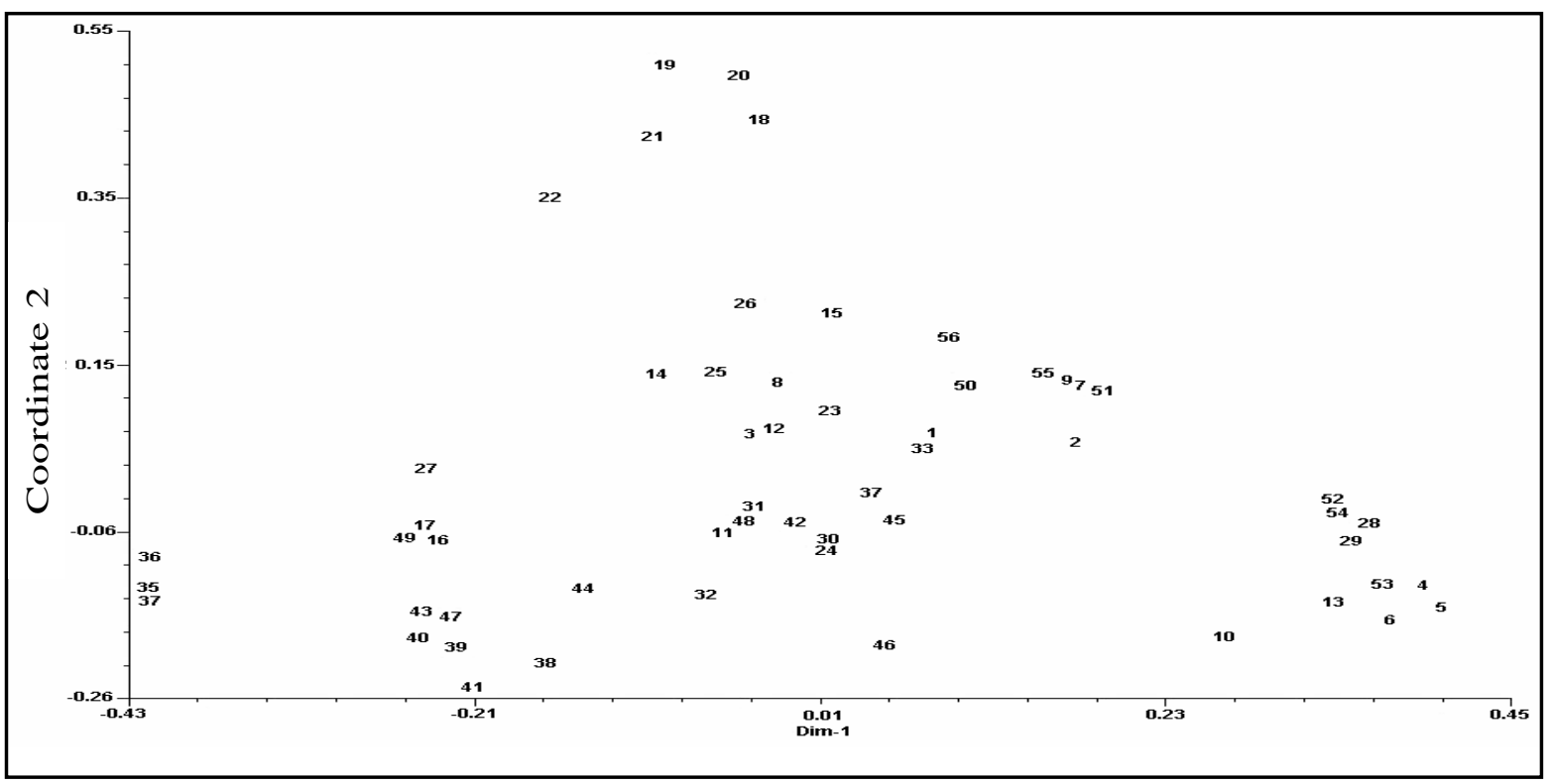

Coordinate 1

Figure 3 - Principal coordinates dispersion graph obtained from DICE genetic similarity matrix, estimated by RAPD markers, between 37 species in the genus Catasetum including 56 individuals, according to the sequence presented in table 3. Londrina (PR, Brazil), UEL, 2004.

It was possible to visualize that cluster data based on DICE's genetic similarity matrix (Table 3) showed interspecific genetic similarity index values ranging from $37 \%$ between Ctsm. laminatum and Ctsm. denticulatum to $83 \%$ (81\% Bootstrap) between Ctsm. triodon and Ctsm. uncatum. Species represented by more than one individual (Table 1) showed an intraspecific genetic similarity index ranging from $88 \%(82 \%$
Bootstrap) for the individuals of the species Ctsm. triodon to $93 \%$ (100\% Bootstrap) between individuals in the Ctsm. atratum group and Ctsm. macrocarpum.

In the principal coordinates dispersion graph obtained from DICE genetic similarity matrix, it was observed that the orchid species under study obeyed almost the same association pattern obtained in the dendrogram, they were distributed 
according to the clusters formed (Fig. 2 and 3). The associations observed in the dendrogram allowed the identification of 11 distinct groups; some groups were formed by species that shared morphological characteristics or occupied the same geographical area.

In the first group, the species Ctsm. aripuanense, Ctsm. Arietinum, and Ctsm. Barbatum were associated with a similarity index of $71 \%(58 \%$ bootstrap), and within this group, two Ctsm. barbatum plants (Ctsm. barbatum semicuculatum and Ctsm. barbatum sp.) were associated with 94\% (100\% bootstrap) similarity. The two plants were collected from distinct geographical regions (Minas Gerais and Amazonas). The ecological conditions in these two Brazilian states are distinct: the State of Minas Gerais (MG) presents a somewhat subtropical hot climate, while the State of Amazonas (AM) presents a more tropical climate with little temperature variation throughout the year and a short dry season with high humidity during the night (Holst, 1999). The climatic differences between both the collection sites and their high genetic similarity value (94\%) suggested that Ctsm. barbatum semicuculatum and Ctsm. barbatum could have split not long ago in evolutionary terms, or that these differences were due to variation in the habitats only.

The species Ctsm. arietinum is very common in its region of origin living under palm trees. Characteristically, its pseudobulbs are arched downward and leaves are longer than usual for the genus. These characteristics are kept constant during cultivation (Holst, 1999). Based on the quantity of cilia on the labellum, Ctsm. arietunum immediately evokes the species Catasetum barbatum, justifying the association herein observed. Differently from this group, however, the flowers are arranged strongly clustered at the distal section in relation to the base. The shape of the labellum is also completely different from other species in the group cited above, as well as the color of its flowers. Another characteristic is that the dispersion area of Ctsm. arietinum is specifically located in the Brazilian Northeastern region (PE), where other species of this previously-mentioned complex cannot be found. In this genus, the only other species confirmed in the region was Ctsm. discolor Lindl. (ABRACC, 1995), but it was not associated with this group, which showed a CS of 54\% with Ctsm. arietinum. The species Ctsm. arietinum is described in the literature as belonging to the Ctsm. cristatum complex (Holst, 1999). However, in this study, Ctsm. cristatum appeared associated with another group, presenting a genetic similarity of only $60 \%$ with Ctsm. arietinum. In spite of the fact that they have ciliated labella, which was a distinctive trait, these species were not linked in the Dendrogram. This could have happened due to the fact that both species have distinct geographical distributions, with Ctsm. arietinum distributed mainly in the State of Pernambuco, while Ctsm. cristatum is distributed across the States of Roraima, Amapá, and Pará. However, although these species have different concentration areas, they share the same ecological climate distribution, consisting of a hot, humid, and tropical climate which remains unchanged almost the entire year, with high moisture during the night, even during dry periods, which are quite short (Holst, 1999).

In the first group, there was also Ctsm. aripuanense, which was associated with species of Ctsm. barbatum and Ctsm. arietinum, with a genetic coefficient of similarity of $72 \%$. These three species present some similarities, and are found in the States of MT and MS (with a wider distribution for Ctsm. barbatum). They show medium flowering in summer, and their main trait is the presence of numerous cilia on the labellum (Raposo, 1992; Holst, 1999). The second group was associated with a mean similarity of $69 \%$ (44\% Bootstrap) to the species: Ctsm. atratum, Ctsm. cernnum, Ctsm. confusum, Ctsm. hookeri, Ctsm. triodon, and Ctsm. uncatum. In this group, the intraspecific genetic similarity was high (93\%), with $100 \%$ bootstrap between Ctsm. atratum plants. The species Ctsm. atratum can be found in the states of SP, PR, SC, RS, and MG (Raposo, 1992); it occurs in mountainous regions under common subtropical climate ecological conditions (Holst, 1999), which may justify their high genetic similarity value.

Ctsm. cernnum and Ctsm. confusum were associated with $78 \%$ similarity (bootstrap=70). Although these two species are not morphologically alike, clustering may have occurred because both Ctsm. cernuum and confusum present concave petals and sepals with dark red spots, medium flowering in summer, and an epiphytic growth habit (Holst, 1999). The species Ctsm. triodon and Ctsm. uncatum were associated with $82 \%$ similarity (bootstrap $=82$ ). However, these species present morphological differences and cannot be found in the same geographical region. Ctsm. triodon is found in the 
States of SC, PR, and RS, while Ctsm. uncatum occurs in the States of PE, AL, CE, and BA.

A $3^{\text {rd }}$ group to become associated involved Ctsm. ariquimense, Ctsm. lemosii, and Ctsm. matogrossense, with a mean similarity of $63 \%$. In general, the species in this group have few morphological similarities, in agreement with the RAPD data. In addition, these species are generally found in distinct geographical regions, except Ctsm matogrossense and lemosii, which can be found in MT, submitted in this case to the same ecological conditions, thus justifying the higher similarity between these species (CS= 67\%). Catasetum ariquimense could be linked to Ctsm. barbatum due to the presence of fimbriae; representatives of the barbatum complex, however, do not occur in the same geographical region as Ctsm. ariquimense (ABRACC, 1996). These two species obtained a coefficient of similarity of $54 \%$, reinforcing the responses obtained in this experiment.

The fourth group to become linked, with a mean similarity of $93 \%$, was the group containing Ctsm. fimbriatum species (bootstrap=92). Ctsm. fimbriatum with dark red flowers was a little more isolated than the others. Because this color is not very common in this species, it is suggested that it could be a mutation; therefore, it could be a new variety.

The species Ctsm. galeritum, Ctsm. spitzii, and Ctsm. vinaceum were associated in the fifth group, with a mean similarity of $70 \%$. These three species can be found in the State of GO; however, Ctsm. galeritum and Ctsm. vinaceum have a wider geographical distribution. Catasetum pulchrum shows few morphological similarities with the other two species in the group, having differentiated flowers, particularly due to brown stripes disposed across petals and sepals on a light background (Holst, 1999). Catasetum pulchrum was associated with species in this group with a mean similarity of $61 \%$.

Although associated with the species Ctsm. spitzii and Ctsm vinaceum, Ctsm. galeritum did not present common morphological characteristics either. However, this species can bloom in the same season as Ctsm. spitzii and can also be found under the same climatic and ecological conditions of occurrence of the species Ctsm. spitzii and Ctsm. vinaceum. These climatic and ecological conditions are represented by an essentially continental climate, where drought periods are long and moisture is very low through almost the whole year, and days are hot and dry, while nights are reasonably cold (Holst, 1999).

The association demonstrated in the dendrogram (mean similarity of $70 \%$ ) between the species Ctsm. spitzii and Ctsm. vinaceum confirmed what had been previously described in the literature. According to Holst (1999) and ABRACC (1996), greater affinity was found between the species Ctsm. spitzii and the species Ctsm. vinaceum and Ctsm. trulla Lindl. (not used in this experiment). However, Ctsm. spitzii has a more convex than sack-shaped labellum on its male flowers, having only a light depression below the middle, and long fleshy bristles on the margins of the lower half. According to Holst (1999), both species are morphologically similar at the vegetative stage, and discrimination can only be made after flowering.

The $6^{\text {th }}$ group clustered Ctsm cristatum, Ctsm. shimidtianum, Ctsm. discolor, Ctsm. gnomus, and Ctsm. moreanum species with a mean similarity of $67 \%$. These data are in agreement with the small morphological similarity among these species. According to Miranda and Lacerda (1992), while the genus Catasetum presented taxonomic difficulties, some of its representative species, including Ctsm. cristatum Lindl., were even more complicated due to the apparent similarity of their flowers, both male and female. A knowledge about their geographical distribution has been a facilitating factor in understanding the group, and at present, many species can be clearly defined.

Catasetum cristatum is the northernmost of these species in Brazil, occurring from Venezuela to the States of Roraima, Amapá, and Pará. Ctsm barbatum (Lindl.) Lindl. presents the largest dispersion area, along the Solimões, Negro, and Amazonas Rivers and their tributaries in a region with elevations lower than $300 \mathrm{~m}$, until the coastal region with higher-moisture microclimates of the Brazilian Northeast. Species related to this genus are found in the States of Minas Gerais and São Paulo; however, these are still under study, since descriptions already made for plants in this region present many controversies. Some species and varieties described in the $19^{\text {th }}$ century had their types destroyed and were described in little detail, with not very enlightening drawings, and vague references to the site of origin, which prevented them from being validated and making it difficult to correlate what had been published with the present knowledge (ABRACC, 1998). From this work, it could be, therefore, concluded that the 
species in the Ctsm. cristatum complex were distinct species, since they clustered separately.

The $7^{\text {th }}$ group clustered the species Ctsm. macrocarpum, Ctsm. osculatum, and Ctsm. saccatum. The intraspecific level of variation for Ctsm. macrocarpum was $93 \%$ (100\% bootstrap), and $84 \%$ for Ctsm. osculatum. The mean genetic similarity among the three species was $71 \%$. However, the species Ctsm macrocarpum was morphologically different from the species Ctsm. saccatum and Ctsm. osculatum, which were morphologically more similar. Two of the analyzed Ctsm. macrocarpum plants were collected in the States of PA and BA; the geographical distribution of this species is very wide, comprising the States of RJ, BA, PB, PE, RN, PI, PA, AM, TO, MT, MA, RR, and ES. The ecological conditions in these states are more or less similar, with the climate practically identical throughout the year, with temperature ranging from $30^{\circ} \mathrm{C}$ to $15^{\circ} \mathrm{C}$ (Holst, 1999). Because they were found in nearby regions and under the same ecological condition, the high CS value found (93\%) was justified.

Within the same group, the species Ctsm. saccatum and Ctsm. osculatum were clustered with $69 \%$ genetic similarity. Although this similarity was not very high, these species had a lot in common, but were also different in many points, justifying the CS value found in this experiment. According to ABRACC (1996), the morphological differences between Ctsm. osculatum and Ctsm. saccatum are as follows:

-Ctsm. osculatum presents a flower stalk that begins erect but becomes arched under the weight of the flowers, while Ctsm. saccatum presents a pendant flower stalk;

-The male and female flower pedicels in Ctsm. osculatum are up to $3.5 \mathrm{~cm}$ in length, while in Ctsm. saccatum they have a length of up to 6.2 $\mathrm{cm}$;

-Male flowers in Ctsm. osculatum are smaller and usually yellowish-green or greenish-brown in color, with small spots when present, while Ctsm. saccatum has larger, dark, brown or reddish-brown flowers, always presenting evident, larger spots;

-The floral elements proportions in both species are significantly different: Ctsm. saccatum has long and narrow sepals and petals, while in Ctsm. osculatum they are short and broad;

-The labellum in Ctsm. osculatum is subcordate (heart-shaped) while in Ctsm. saccatum it is trilobate. The labellum in Ctsm. osculatum is broader than long;

-Female flowers in Ctsm. osculatum are much smaller than in Ctsm. saccatum;

The $8^{\text {th }}$ group associated the species Ctsm. gladiatorum and Ctsm. juruenense with 65\% similarity; these apparently did not have much in common beyond the absence of female flowers and the presence of cilia on the labellum, which were, however, much smaller in size in Ctsm. juruenense (Raposo, 1992; ABRACC, 1996; Lacerda, 1998 a, b; Holst, 1999).

Catasetum gladiatorium is distributed across a wide area in the Brazilian Central Plateau, in the States of Mato Grosso, Goiás, and Tocantins, and is epiphytic in palm trees that occur in regions under artificial pastures and cerrados, at elevations between 230 and $790 \mathrm{~m}$. The species emits two to three consecutive flower stalks per year and blooms in the summer and fall. The species Ctsm. spitzii, Ctsm. fimbriatum, Ctsm. rooseveltianum, Ctsm. galeritum, Ctsm. fuchsii, Ctsm. ornithoides, Ctsm. osculatum, Ctsm. schmitdianum, and Ctsm. vinaceum also occur in the region (Lacerda, 1998 a, b). Catasetum gladiatorum showed a 63\% coefficient of similarity with Ctsm. cristatum, and has been considered a member of the cristatum complex in the literature, but has also been previously classified as Ctsm barbatum, with which it obtained $60 \%$ similarity (Table 4 ).

The $9^{\text {th }}$ group included the species Ctsm. parguazense, Ctsm. pileatum, Ctsm. purum and Ctsm. planiceps. The greatest genetic proximity was verified between the species Ctsm. purum and Ctsm planiceps, with a $72 \%$ similarity association. These species presented marked morphological differences and a very distinct geographical distribution. While Ctsm. planiceps can be found in the State of AM (north of Brazil), Venezuela, the Guianas, Suriname, and Peru, Ctsm. purum is found in the States of BA, ES, and MG.

Although Ctsm. purum and Ctsm uncatum were not clustered in the same group in the dendrogram (Genetic coefficient of similarity $=55 \%$ ), they have traits in common that could differ in some points. According to Lacerda (1997) these differences are as follows:

-both have roots, rhizomes, pseudobulbs, and leaves typical of the genus, but Ctsm. purum attains a larger size;

-male flowers are green in both, but a little smaller in Ctsm. uncatum; 
-both species occur in Bahia, with Ctsm Purum extending until Minas Gerais and Espírito Santo, and Ctsm. uncatum extending until Pernambuco. The species Ctsm. pileatum and Ctsm parguazense are considered unique, and do not show many similarities, especially with regard to their flowers in relation to other species in the genus. The species Ctsm. complanatum was displayed individually between groups 9 and 10. This isolation could be due to a not very significant morphological similarity with the other species. Ctsm. complanatum was a species related to Ctsm. cirrhaeoides, which was not used in this experiment. The only differences between them are: the presence of an arched, non-pendulous flower stalk, flowers concentrated at the distal end of the inflorescence (whereas in the other orchid the flowers are located very close to the pseudobulb), a more sharply-pointed and flat conical labellum, and a quite distinct geographical distribution in both species (ABRACC, 1999).

Ctsm. denticulatum is a species that occupies the same geographical and climatic region as Ctsm. complanatum; these species obtained a similarity matrix value of $51 \%$ between themselves, but were not associated in the dendrogram.

The $10^{\text {th }}$ group showing associations contained the species Ctsm. ciliatum, Ctsm. gardineri, and Ctsm. sanguineum. Ctsm. ciliatum and Ctsm. gardineri were associated with 59\% similarity. Ctsm. sanguineum was a little more distant from the group, with 56\% similarity (Table 4).

Ctsm. ciliatum is considered a synonym of Ctsm. roseo-album, which is treated as a variety of Ctsm. discolor. Ctsm. gardineri is also considered a synonym of Ctsm. discolor, with only a few differences, such as smaller and more yellow flowers in the species Ctsm. gardineri than in Ctsm. discolor; the Ctsm. gardineri plant as a whole is more robust (Holst, 1999).

The association between Ctsm. discolor and Ctsm. gardineri and Ctsm. ciliatum was not shown in the dendrogram; however, the three species were associated in the matrix with $69 \%$ similarity; also, Ctsm. discolor and Ctsm. gardineri obtained 66\% similarity, while Ctsm. discolor and Ctsm. ciliatum obtained $65 \%$ similarity. This association showed that the species Ctsm. gardineri and Ctsm. ciliatum were more similar to Ctsm. discolor than between themselves.

In the dendrogram, the species Ctsm. laminatum and Ctsm denticulatum were isolated from other species in the same genus. The isolation observed for Ctsm. laminatum could be due to its geographical distribution; it occurs in Mexico, in the States of Michoacan, Guerrero and Oaxaca, and cannot be found in Brazil as the others (Holst, 1999).

Ctsm denticulatum, together with Ctsm. pulchrum N.E. Brown and Ctsm. cirrhaeoides Hoehne., form a group with pendant and somewhat dense inflorescences. The denticulate margins of the labellum are the most important aspect in discriminating Ctsm. denticulatum from the other two, which have smooth margins (Lacerda, 2001). The species Ctsm. pulchrum and Ctsm denticulatum were not in the same group and obtained a low similarity index of $47 \%$. This low index could be explained because these species came from distinct geographical regions (States of AM, PA, and MT for Ctsm. pulchrum, and RO for Ctsm. denticulatum), and also because they were under different ecological and climatic conditions.

\section{CONCLUSIONS}

These results demonstrated the great genetic diversity of the species analyzed. The high polymorphism index obtained showed that RAPD markers could also aid to determine the relations in the genus Catasetum, in addition to being effective in identifying the polymorphism itself. The information generated based on these data could contribute to define the strategies for the establishment of germplasm collections, as well as to elucidate the problems related to the classification of species. It is still important to emphasize that additional studies with a bigger number of plants and other species would be important and necessary for a better definition of strategies.

\section{ACKNOWLEDGEMENTS}

We are grateful to Conselho Nacional de Desenvolvimento Científico e Tecnológico (CNPq) and to Coordenação de Aperfeiçoamento de Pessoal de Nível Superior (CAPES) for financial support. 


\section{RESUMO}

Neste trabalho, marcadores moleculares de RAPD foram utilizados para acessar a variabilidade genética e estudar as relações interespecíficas e intraespecífica em um grupo de 37 espécies, compreendendo 56 plantas individuais. Um total de 15 primers foram selecionados para amplificação do DNA. De um total de 221 bandas analisadas, 209 (95\%) foram polimórficas. O nível de similaridade genética interespecífica variou de $37 \%$ entre Catasetum complanatum e Catasetum laminatums a $83 \%$ entre Catasetum triodon e Catasetum uncatum. A similaridade genética intraespecífica variou de $88 \%$ entre os indivíduos de Catasetum triodon a $93 \%$ entre os indivíduos de Catasetum atratum e Catasetum macrocarpum. Os resultados deste trabalho contribuem para o entendimento das relações interespecíficas no gênero Catasetum, para definir estratégias para o estabelecimento de um banco de germoplasma e para dar suporte a programas de melhoramento.

\section{REFERENCES}

ABRACC (1995 a 1999), Jornal da ABRACC: Associação de Brasileira de Catasetíneas.

Carnier, A. (1996), Variação no número de cromossomos nas Orquídeas. Jornal da ABRACC: Associação Brasileira de Cultivadores de Catasetíneas, Ano 2, n. 6, Julho.

Case, M.A.; Mlodozeniec, H.T.; Wallace, L.E. and Weldy, T.W. (1998), Conservation genetics and taxonomic status of the rare Kentucky Lady's Slipper: Cypripedium kentuckiense (Orchidaceae). American Journal of Botany, 85, 1779-1786.

Coelho, A.S. (2001), Avaliação de dendrogramas baseado em estimativas de distâncias/similaridades genéticas através do procedimento de bootstrap. V.3.0. Goiânia.

Doyle, J.J. and Doyle. J.L. (1987), A rapid DNA isolation procedure for small quantities of fresh leaf tissue. Phytochemistry Bulletin, 19, 11-15.

Dressler, R. L. (1993). Phylogeny and classification of the orquid family. Theodore R. Dudley (ed.). Oregon: Discords Press Portland. 327.

Faria, R.T.; Rego, L. V. and Bernardi, A. (2001). Performance of Differents Genotyps of Brazilian Orchid Cultivation in Alternative Substrates. Brazilian Archives of Biology and Technology, 44, 337-342.
Freudenstein, J.V. and Doyle, J.J. (1994), Character transformation and relationships in Corallorhiza (Orchidaceae: Epidendroideae). I. Plastid DNA. American Journal of Botany, 81, 1449-1457.

Gravendeel, B.; Chase, M.W.; Vogel, E. F.; Roos, M.C.; Mes, T.H.M. and Bachmann, K. (2001), Molecular phylogeny of Coelogyne (Epidendroideae; Orchidaceae) based on plastid RFLPS, mat $K$, and nuclear ribosomal ITS sequences: evidence for polyphyly. American Journal of Botany, 88, 19151927.

Heywood, V. H. (1993), Flowering plants of the world. London: B T Batsford.

Holst, A.W. (1999), The World of Catasetums. Portland, Oregon, USA: Timber Press.

Lacerda, K.G. (1997), Características diferenciais entre Catasetum purum Nees and Seenings e Catasetum uncatum Rolfe. Jornal da ABRACC: Associação Brasileira de Cultivadores de Catasetíneas., 3 (11), 45.

Lacerda, K.G. (1998a), Catasetum gladiatorium., Bradea,16, 90.

Lacerda, K.G. (1998b), Catasetum gladiatorium. Jornal da ABRACC: Associação Brasileira de Cultivadores de Catasetíneas, ano IV, 16, pp.2-4.

Lacerda, K.G. (2001), Catasetum denticulatum Jornal da ABRACC: Associação Brasileira de Cultivadores de Catasetíneas, ano IV, 16, pp. 2-3.

Miranda, F and Lacerda, K.G. (1992), Catasetum complanatum sp. Nov. Bradea- Boletim do Herbarium, vol. VI, n 7.

Moreira, A.S.F.P. and Isaias, R.M.S. (2008), Comparative anatomy of the absorption roots of terrestrial and epiphytic orchids. Brazilian Archives of Biology and Technology, 51, 83-93.

Obara-Okeyo, P. and Kako, S. (1998), Genetic diversity and identification of cymbidium cultivars as measured by random amplified polymorphic DNA (RAPD) markers. Euphytica, 99, 95-101.

ORCHIDACEAE BRASILIENSIS. Catasetum. Disponível em: < http://www.dalholl.hpg.ig.com.br/generos/Catasetum/ Catasetum.html> Acesso18.fev.2004.

Pridgeon, A.M.; Solano, R. and Chase, M.W. (2001), Phylogenetic relationships in Pleurothallidinae (Orchidaceae): combined evidence from nuclear and platid DNA sequences. American Journal of Botany, 88, 2286-2308.

Raposo, J. G. (1992), A Etimologia a Serviço dos Orquidófilos- Vol. I. Editora Ave-Maria- São Paulo.

Rittershausen, V. (1998), O Maravilhoro mundo das Orquídeas. Ed Salamandra, Rio de Janeiro-RJ.

Rohlf, F.J. (2000), Statistical power comparision among alternative morphometric methods. American Journal of Physics Anthropology, 111, 463-478. 
Scaglia, J.A.P. (1998), Como calassificar corretamente um Catasetum. O mundo das Orquídeas. 4, pp.7-8.

Suttleworth, F.S.; Zim, H.S. and Dillon, G.W. (1993), Orquídeas: Guia dos orquidófilos. Rio de Janeiro: Expressão e Cultura.

Squirrell, J.; Hollingsworth, P.M.; Bateman, R.M.; Dickson, J.H.; Light, M.H.S.; Macconaill, M. and Tebbitt, M.C. (2001), Partitioning and diversity of nuclear and organelle markers in native and introduced populations of Epipactis helleborine (Orchidaceae). American Journal of Botany, 88, 1409-1418.

Sun, M. and Wong, K.C. (2001), Genetic structure of three orchid species with contrasting breeding systems using RAPD and allozyme markers. American Journal of Botany, 88, 2180-2188.

Tanaka, R. and Kamemoto, H. (1984), Chromosomes in the orchids: counting and numbers. In:. ARDITTI, J. Orchid Biology: Reviews and Perpectives, III. Ithaca, New York: Cornell University Press, pp. 323410 .
Tsai, C. C., Huang, S. C., Huang, P. L., Chen, Y. S. and Chou, C. (2002), H..Phenetic relationship and identification of subtribe Oncidiinae genotypes by random amplified polymorphic DNA (RAPD) markers. Scientia Horticulturae, 96, 303-312.

Williams, N. H.; Chase, M.W. and Whitten, M. (2001), Phylogenetic positions of Miltoniopsis, Caucae, a new genus, Cyrtochiloides, and Oncidium phymatochilum (Orchidaceae: Oncidiinae) based on nuclear and platid DNA sequence data. Lindleyana, 16, 272-285.

Received: May 17, 2007; Revised: February 26, 2008; Accepted: May 05, 2009. 